\title{
Benchmarking com foco na satisfação dos usuários do transporte coletivo por ônibus
}

\section{Mariana Müller Barcelos ${ }^{1}$, Luis Antonio Lindau², Maria Beatriz Berti da Costa ${ }^{3}$, Carla ten Caten ${ }^{4}$, Cristina Albuquerque ${ }^{5}$, Brenda Medeiros Pereira ${ }^{6 \bowtie}$}

${ }^{1}$ WRI Brasil Cidades Sustentáveis, mariana.barcelos@wri.org

${ }^{2}$ WRI Brasil Cidades Sustentáveis, toni.lindau@wri.org

3Departamento de Engenharia de Produção e Transportes, UFRGS, beacosta@hotmail.com

${ }^{4}$ Departamento de Engenharia de Produção e Transportes, UFRGS, tencaten@producao.ufrgs.br

${ }^{5}$ WRI Brasil Cidades Sustentáveis, cristina.albuquerque@wri.org

6WRI Brasil Cidades Sustentáveis, brenda.medeiros@wri.org

\section{Recebido:}

20 de fevereiro de 2017

Aceito para publicação:

27 de setembro de 2017

Publicado:

29 de outubro de 2017

Editor de área:

Cira Souza Pitombo

\section{Palavras-chaves:}

Benchmarking,

Pesquisa de satisfação,

Qualidade com foco no usuário,

Transporte coletivo por ônibus,

Normalização.

\section{Keywords:}

Benchmarking,

Satisfaction survey,

Quality focused on users,

Bus public transport,

Normalyzing.

DOI:10.14295/transportes.v25i3.1335
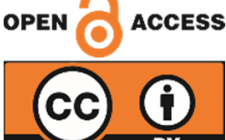

\begin{abstract}
RESUMO
Investir na qualidade do transporte coletivo é fundamental para fidelizar e atrair usuários e fomentar cidades mais sustentáveis. O benchmarking é uma ferramenta adequada para identificar melhores práticas e promover troca de experiências focadas na melhoria dos sistemas de transportes. No entanto, o benchmarking com foco na satisfação dos clientes impõe desafios devido à falta de padronização na coleta de dados e aos vieses socioculturais inerentes a pesquisas de opinião. Este trabalho apresenta uma análise de benchmarking a partir de dados de satisfação, coletados através de uma pesquisa padronizada. Propõe-se uma normalização das notas de satisfação que: (i) reduz o efeito dos vieses socioculturais, (ii) possibilita a comparação de sistemas de ônibus que operam em distintas cidades brasileiras, e (iii) proporciona a identificação de potenciais benchmarks. O método proposto revelou-se adequado na identificação de metas, prioridades e atributos de sistemas de transporte coletivo por ônibus que podem servir de referência para outras cidades.
\end{abstract}

\begin{abstract}
Investing in quality of public transportation is fundamental to keep and attract users and to foster more sustainable cities. Benchmarking is an adequate tool to identify best practices and promote exchange experiences that contributes to improve transportation systems. Nevertheless, benchmarking focused on client satisfaction imposes challenges because of the lack of standards in collecting data and the socio-cultural biases inherent to opinion surveys. This paper presents a benchmarking analysis based on satisfaction data collected through a standardized survey. We propose a normalization of satisfaction rates that: (i) reduces the effect of socio-cultural biases, (ii) enables the comparison of bus systems operating in different Brazilian cities, and (iii) allows the identification of potential benchmarks. The proposed method proved suitable in identifying goals, priorities and attributes of bus transit systems that can serve as reference to other cities.
\end{abstract}

\section{INTRODUÇÃO}

A qualidade do transporte público ganhou relevância em muitas cidades nos últimos anos. Um dos desafios é compreender como o serviço impacta os usuários e o que deve ser feito para melhorar a oferta. Assim, investir em ferramentas que sejam capazes de traduzir de maneira clara e objetiva as necessidades e percepções dos usuários passa a ser essencial para que as ações sejam mais efetivas.

O benchmarking é uma ferramenta de qualidade disseminada globalmente. Consiste em um processo de comparação e troca de experiências com o objetivo de identificar boas práticas e soluções para a melhoria da qualidade das organizações (American Productivity \& Quality Center, 1996). Tipicamente, o 
benchmarking é usado para melhorar os processos e reduzir custos. Recentemente, aspectos relacionados ao cliente também surgiram dentro dos grupos de benchmarking. Na área de transporte coletivo não é diferente, a percepção dos usuários é abordada pelo International Bus Benchmarking Group (IBBG) e Benchmarking European Service of Public Transport (BEST) (Trompet et al., 2013; EMTA, 2016), em que diferentes sistemas de transporte aplicam pesquisas padronizadas de satisfação online.

0 presente trabalho aborda o benchmarking com enfoque na satisfação dos usuários do serviço de transporte coletivo por ônibus no Brasil. Para mensurar a percepção dos usuários, foi utilizada a Pesquisa de Satisfação Qualiônibus, desenvolvida pelo WRI Brasil. Trata-se de uma pesquisa padronizada que vem sendo aplicada em cidades brasileiras para mensurar o nível de satisfação e percepção dos usuários em relação aos sistemas de transporte coletivo por ônibus (EMBARQ Brasil, 2014).

A comparação de pesquisas de satisfação, aplicadas em diferentes locais, é um desafio devido às diversas diferenças entre tipos de pesquisas, questionários, amostras e contextos culturais e sociais (Trompet et al., 2013). Enquanto os primeiros itens podem ser solucionados com a aplicação de uma pesquisa padronizada, aspectos socioculturais são inerentes a pesquisas de opinião e precisam ser relativizados para tornar as diferentes aplicações comparáveis.

Assim, este estudo propõe uma normalização das notas de satisfação dos usuários de transporte coletivo com a finalidade de reduzir os vieses culturais e sociais. Dessa forma, torna-se possível realizar a comparação entre cidades para identificar aquelas que têm potencial de ser referência de boas práticas e permitir que o processo de benchmarking seja realizado com dados de satisfação.

Inicialmente, é apresentada uma revisão da literatura sobre a qualidade com foco no cliente e processos de benchmarking, seguida da descrição do método empregado. Após, são apresentadas as análises de benchmarking realizadas e, por fim, são apresentadas as considerações finais.

\section{A QUALIDADE COM FOCO NO CLIENTE}

Historicamente, o transporte público urbano teve a qualidade associada a aspectos operacionais do sistema, traduzindo predominantemente os interesses dos operadores e dos órgãos gerenciadores do sistema de transporte público. Foi a partir da década de 1990 que o conceito de qualidade passou a ser utilizado mais amplamente, agregando também as visões pela percepção dos usuários, incluindo os desejos e as necessidades da sociedade (Bertozzi e Lima Jr., 1998). Ainda assim, grande parte dos gestores de transporte ainda não iniciou este processo. Introduzir iniciativas de medição da percepção dos usuários nos processos de gestão acaba sendo um diferencial adotado por órgãos gerenciadores com mais recursos e também com usuários que possuem uma diversidade maior de opções de transporte (TCRP, 2003a).

O ciclo da qualidade, proposto pela Norma Europeia EN 13816, permite entender quais são os componentes envolvidos na qualidade do transporte coletivo. Conforme ilustrado na Figura 1, no âmbito dos clientes do transporte e da comunidade, a medida de satisfação dos usuários é definida pela diferença entre a qualidade desejada e a percebida do serviço. Já nas agências e operadores do transporte, a medida de desempenho é determinada pela diferença entre a qualidade de serviço contratada e a ofertada (European Standard, 2002). As diferenças entre os quatro tipos de qualidade são denominadas gaps da qualidade. Almeja-se que esses gaps sejam os menores possíveis. Este ciclo pode ser utilizado como uma ferramenta de gerenciamento, tanto no âmbito da rede de transporte coletivo, abrangendo a área urbana, quanto de uma linha ou rota de um sistema (European Comission, 1998).

A qualidade na área de transporte coletivo deve ser definida como a qualidade pelo ponto de vista do passageiro (Kittelson \&Associates, 2003), já que ela deve ser entendida como instrumento para atrair novos usuários ao transporte e manter os que já utilizam. Assim, o usuário deve ser visto como cliente do sistema de transporte, tendo direito a um serviço de boa qualidade e motivos para que continue usando o sistema de transporte público, considerado o mais sustentável para as cidades (Ferraz e Torres, 2004). 0 presente trabalho tem foco na "Medida de Satisfação", gap entre "Qualidade desejada" e "Qualidade percebida" do Ciclo da Qualidade. 


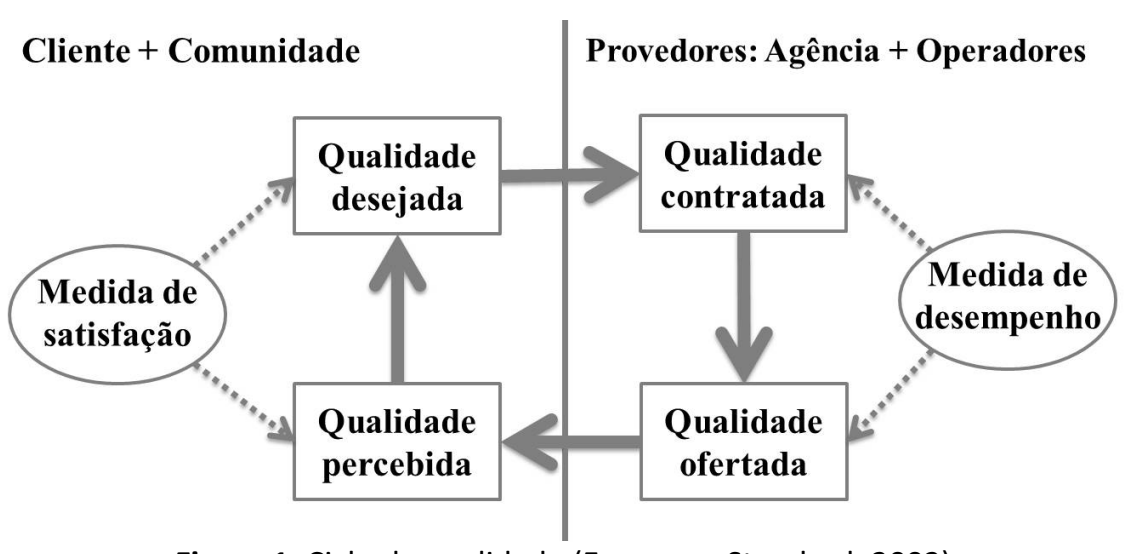

Figura 1. Ciclo da qualidade (European Standard, 2002)

\section{O PROCESSO DE BENCHMARKING}

O benchmarking é uma ferramenta que tem como objetivo revelar as melhores práticas de uma organização reconhecida como competente em determinado aspecto e tê-la como referencial para promover melhorias no desempenho das demais (Butta e Huq, 1999). 0 benchmarking teve início em 1979, quando a Xerox Corporation, após sofrer uma grande redução do mercado que detinha, aplicou o processo pela primeira vez, disseminando a prática no âmbito global (Zairi, 1996).

0 processo de benchmarking consiste em reconhecer as falhas da própria organização, reconhecer que alguém esteja fazendo um trabalho melhor, aprender como ele está sendo feito e, em seguida, implementá-lo (American Productivity \& Quality Center, 1996). Assim, o benchmarking permite um foco externo e obriga olhar para o que seus concorrentes estão fazendo (Huq e Bhutta, 1999). Os grupos de benchmarking geralmente se apoiam em uma política de sigilo e anonimato das informações disponibilizadas, o que garante a total abertura dos dados dentro do grupo e confidencialidade para o exterior (Randall et al., 2007).

Em transportes, diversos grupos de benchmarking nacionais e internacionais foram criados para melhorar a qualidade dos serviços, como Community of metros - CoMET, International Bus Benchmarking Group - IBBG, Suburban Rail Benchmarking Group - ISBeRG, American Bus Benchmarking Group - ABBG, Benchmarking European Service of public Transport - BEST, Grupo de Benchmarking Fetranspor (Federação das Empresas de Transportes de Passageiros do Estado do Rio de Janeiro). 0 foco desses grupos reside, em sua maior parte, no desempenho e custos operacionais dos sistemas de transporte (Randall et al., 2007; Ribeiro e Gonçalves 2015, Alouche e Barbosa, 1997), muito embora aspectos relacionados à satisfação dos clientes tenham surgido em alguns deles recentemente (Trompet et al., 2013; EMTA, 2016).

O benchmarking baseado na satisfação do usuário é um desafio devido às diferenças entre tipos de pesquisas, questionários, amostras, contextos sociais e políticos, etc. (Trompet et al., 2013). Mesmo quando se padroniza a metodologia de aplicação das pesquisas, corre-se o risco de ter as respostas dos usuários com um viés cultural e social que pode, inclusive, invalidar comparações entre diferentes países ou cidades de um mesmo país (Morpace International e Cambridge Systematics, 1999; EQUIP Consortium, 2000).

Devido à importância da satisfação do cliente, em 2009, os membros do International Bus Benchmarking Group (IBBG) incluíram análises de satisfação utilizando uma metodologia de normalização de dados para permitir o benchmarking da satisfação do cliente. 0 objetivo seria não comparar diretamente a satisfação dos clientes entre as diferentes cidades, mas sim normalizar os resultados da satisfação para entender o quanto a qualidade dos sistemas está atendendo às expectativas dos clientes nas diferentes áreas do serviço prestado (Trompet et al., 2013). 


\section{MÉTODO ADOTADO}

A realização de análises de benchmarking com foco na percepção dos usuários pressupõe a coleta de informações de forma padronizada. No Brasil, não há disseminação nacional de um mesmo procedimento envolvendo amostragem, questionário, forma de aplicação e análise. Para que seja possível comparar os resultados de diferentes cidades brasileiras utilizou-se dados coletados pelo WRI Brasil através da Pesquisa de Satisfação Qualiônibus.

0 método proposto para o benchmarking apoia-se em uma extensa base de dados. Após avaliar a representatividade das amostras, o presente trabalho utiliza um método de normalização das notas de satisfação para superar os vieses socioculturais e possibilitar a comparação entre as cidades. Nesta seção apresenta-se a pesquisa utilizada e os procedimentos metodológicos adotados para realizar o benchmarking.

\subsection{Pesquisa de satisfação}

A Pesquisa de Satisfação Qualiônibus mede a percepção de usuários do transporte coletivo por ônibus e foi concebida e aplicada em cidades brasileiras pelo WRI Brasil (EMBARQ Brasil, 2014). Foi desenvolvida com base em extensiva revisão da literatura internacional (como relatórios do Transit Cooperative Research Program - TCRP, Norma Europeia 13826, entre outros) e de pesquisas aplicadas no Brasil e internacionalmente (como as aplicadas pelo Transantiago, TransMilenio, Consórcio Transportes Madrid, Urbanização de Curitiba - URBS, São Paulo Transporte S.A. - SPTrans, Empresa de Transportes e Trânsito de Belo Horizonte - BHTRANS, Associação Nacional de Transportes Públicos - ANTP, entre outros).

Embora padronizada, a pesquisa conta com flexibilidade para se adequar aos diversos contextos de cidades. A mensuração da percepção dos usuários é utilizada, posteriormente, para apoiar a tomada de decisão, verificar o impacto de intervenções realizadas no sistema de transporte coletivo e apoiar o processo de gestão da qualidade (EMBARQ Brasil, 2014). Os dados utilizados neste trabalho foram extraídos da aplicação da Pesquisa de Satisfação Qualiônibus em quatro cidades brasileiras.

\subsubsection{Questionário}

O questionário da Pesquisa de Satisfação Qualiônibus é composto por um módulo básico e diversos módulos detalhados. 0 módulo básico é obrigatório a todas as cidades que desejam aplicar a pesquisa, já os detalhados são optativos e podem ser escolhidos pela cidade dependendo de suas necessidades e interesses em investigar algum aspecto específico do sistema de transporte (EMBARQ Brasil 2014).

0 módulo básico consiste em: (i) perfil do cliente; (ii) perfil de uso; (iii) satisfação com o sistema, sendo uma pergunta da satisfação geral e dezesseis específicas para cada fator da qualidade; e (iv) concordância com oito afirmações de percepção geral do sistema de transporte. Os dezesseis fatores da qualidade presentes na pesquisa são (EMBARQ Brasil, 2014):

- acesso ao transporte: facilidade de chegar aos pontos de acesso e circular nas estações e terminais;

- disponibilidade: intervalo entre os ônibus, nos horários e locais em que necessito;

- rapidez;

- confiabilidade: chegada no horário previsto;

- facilidade de fazer integrações entre linhas de ônibus e outros modos de transporte, para chegar ao destino;

- conforto dos pontos de ônibus: iluminação, proteção, limpeza, quantidade de pessoas;

- conforto das estações: iluminação, proteção, limpeza, quantidade de pessoas;

- conforto dos terminais: iluminação, proteção, limpeza, quantidade de pessoas;

- conforto dos ônibus: iluminação, limpeza, quantidade de pessoas, assentos; 
- atendimento ao cliente: respeito, cordialidade e preparo dos motoristas, cobradores, funcionários e central de atendimento;

- informação ao cliente: sobre linhas, horários e outras informações;

- segurança pública contra roubos, furtos e agressões no caminho e dentro dos ônibus;

- segurança em relação a acidentes de trânsito;

- exposição a ruído e poluição gerados pelos ônibus;

- facilidade em pagar o ônibus e recarregar o cartão de transporte; e

- gasto com transporte coletivo por ônibus.

\subsubsection{Amostragem}

A Pesquisa de Satisfação Qualiônibus indica o número mínimo de questionários aplicados através de amostragem aleatória estratificada proporcional (Oliveira e Grácio, 2005), utilizando nível de confiança de $95 \%$ e erro amostral máximo de 5\%. No caso de sistemas que possuem mais de 20 mil passageiros diários, são necessárias aproximadamente 400 entrevistas válidas e distribuídas aleatoriamente em todo o sistema (TCRP, 1999). As cidades utilizadas neste trabalho adotaram amostras maiores, o que reduz o erro amostral e permite fazer estratificações dos resultados.

Para garantir a distribuição aleatória das pesquisas no sistema, são calculadas cotas da amostra por linha de ônibus e por faixa-horária do dia (pico manhã, entre pico e pico tarde). A amostra é distribuída nas cotas de forma proporcional a demanda de cada linha, devendo-se garantir que as linhas selecionadas representem pelo menos $90 \%$ da demanda do sistema a ser pesquisado. Do mesmo modo, a distribuição por faixa-horária é realizada de forma proporcional a demanda do sistema em cada uma das faixas-horárias e o período total de pesquisa também deve contemplar 90\% da demanda do sistema (TCRP, 1999).

\subsubsection{Notas de satisfação}

As análises de benchmarking tomaram por base apenas as questões de satisfação dos dezesseis fatores da qualidade do módulo básico da pesquisa de satisfação. Como na coleta dos dados é utilizada uma escala Likert de 5 pontos (Likert, 1932) para facilitar as respostas, ela é posteriormente transformada em notas de 0 a 10. A escala Likert e a respectiva transformação para nota é apresentada na Figura 2.

$\begin{array}{cccccc}\text { Escala Likert } & \begin{array}{c}\text { Muito } \\ \text { insatisfeito }\end{array} & \text { Insatisfeito } & \begin{array}{c}\text { Nem satisfeito, } \\ \text { nem insatisfeito }\end{array} & \text { Satisfeito } & \begin{array}{c}\text { Muito } \\ \text { satisfeito }\end{array} \\ \text { Nontos } & -1- & -2- & -3- & -4- & -5- \\ \text { Notas 0-10 } & 0 & 2,5 & 5,0 & 7,5 & 10\end{array}$

Figura 2. Escala Likert e respectivas notas utilizadas nas questões de satisfação da Pesquisa de Satisfação QualiÔnibus

\subsection{Análises estatísticas}

Inicialmente realizou-se o cálculo do coeficiente de variação das respostas nas diferentes cidades estudadas para representar a dispersão das notas dos respondentes para cada cidade. Por se tratar de uma pesquisa amostral, faz-se necessária a aplicação de análises estatísticas para validar os resultados obtidos. Neste trabalho, aplicou-se Análise de Variância para dois fatores (TWO WAY ANOVA) que permite estudar o efeito dos "Fatores da qualidade" e das "Cidades". A análise teve como objetivo verificar se existem diferenças significativas das notas em pelos menos alguns dos fatores da qualidade estudados e também se existiu diferença em pelo menos uma das quatro cidades. Caso a ANOVA comprove que existem diferenças significativas, é necessária a complementação da análise pela Comparação Múltipla de 
Média (CMM) para conferir quais dos fatores da qualidade e das cidades diferem significativamente entre si, ou seja, quais as diferenças entre notas que podem ser extrapoladas para toda a população da cidade.

\subsection{Normalização da satisfação}

Caso haja diferenças significativas entre as cidades, comprova-se estatisticamente que a avaliação média da percepção dos fatores é diferente para cada cidade, caracterizando um viés entre cidades. Tal viés deve ser eliminado para que não influencie a análise de benchmarking dos diferentes fatores da qualidade. 0 International Bus Benchmarking Group (Trompet et al., 2013) propõe a normalização das notas de satisfação em função da média de notas das cidades. 0 processo de normalização consiste em diminuir da nota de satisfação absoluta (nota de 0 a 10), a média das notas dos fatores da qualidade da própria cidade, conforme apresentado na Equação 1:

$$
N n_{i j}=N a_{i j}-\frac{\sum_{i=1}^{n} N a_{i j}}{n}
$$

em que: $N n_{i j}$ : nota normalizada do fator da qualidade $i$ na cidade $j$;

$N a_{i j}$ : nota absoluta do fator da qualidade $i$ na cidade $j$; e

$n$ : número de fatores da qualidade avaliados na cidade $j$.

\subsection{Análises de benchmarking}

As análises de benchmarking devem possibilitar o reconhecimento de cidades que podem ser referência de boas práticas para as demais e auxiliar na definição de metas e prioridades de melhorias para as cidades. Os dados provenientes da pesquisa de satisfação e a normalização proposta pela Equação 1 permitem realizar essas análises para dados de satisfação. A normalização possibilita comparar as diferentes cidades e identificar as cidades referência, bem como definir metas a serem atingidas para os diferentes fatores da qualidade. Já a definição das prioridades pode ser realizada pelas notas absolutas, de 0 a 10, de satisfação. Ao fim do trabalho é apresentado um exemplo em que essas análises são aplicadas para ilustrar o processo de benchmarking.

\section{RESULTADOS E ANÁLISES}

As análises de benchmarking basearam-se nos resultados da Pesquisa de Satisfação Qualiônibus, realizada junto a quatro cidades brasileiras de grande e médio porte no ano de 2015. Nas cidades C e D, a pesquisa foi aplicada em todo o sistema de transporte coletivo por ônibus da cidade, já nas cidades A e B a aplicação se deu apenas em corredores específicos do sistema. As diferenças de abrangência das aplicações, bem como as particularidades de cada cidade e sistema, devem ser consideradas nas análises dos resultados. Conforme a política de anonimato do benchmarking as cidades não são identificadas. A seguir são apresentadas análises de variabilidade amostral e de benchmarking aplicadas aos dados obtidos.

\subsection{Caracterização da amostra}

Para entender melhor a variabilidade do público com que se trabalha nas cidades, realizou-se a análise do coeficiente de variação da amostra. Verificou-se que a amostra em todas as cidades possui dispersão classificada como alta (maior do que 30\%), o que era esperado por se tratar de pesquisas de opinião com um público tão abrangente como usuários do transporte coletivo. A Tabela 1 apresenta a amostra realizada em cada uma das cidades em que a pesquisa foi aplicada, respectivos erros amostrais, médias das notas de satisfação e coeficientes de variação.

Nota-se que os coeficientes de variação das amostras são semelhantes para as quatro cidades e se 
mantiveram em torno de 50\%. Conhecendo-se a variabilidade real, pode-se recalcular a amostra (Ribeiro et al., 2001), resultando em uma amostra mínima necessária de 384 pesquisas (para amostra mínima, considera-se 1 agrupamento, caso em que não serão realizadas análises estratificadas). A amostra mínima recalculada valida a amostra proposta na metodologia da Pesquisa de Satisfação Qualiônibus, que define aproximadamente 400 entrevistas para sistemas acima de vinte mil passageiros por dia (conforme citado em 4.1.2).

Tabela 1: Características das amostras para as quatro aplicações da pesquisa de satisfação

\begin{tabular}{lllll}
\hline Cidade & Amostra realizada & Erro amostral real & Média das notas de satisfação* & Coeficiente de variação da amostra** \\
\hline Cidade A & 2.000 & $2,20 \%$ & 5,41 & $48,4 \%$ \\
Cidade B & 2.599 & $1,93 \%$ & 5,73 & $42,1 \%$ \\
Cidade C & 2.012 & $2,19 \%$ & 4,86 & $55,2 \%$ \\
Cidade D & 527 & $4,27 \%$ & 5,16 & $55,5 \%$ \\
\hline
\end{tabular}

* Média das notas de satisfação dos 16 fatores da qualidade da Pesquisa de Satisfação Qualiônibus

** Coeficiente de Variação calculado com base nas respostas de satisfação dos 16 fatores da qualidade

\subsection{Análises de benchmarking através da satisfação dos usuários}

Após aplicação da pesquisa nas quatro cidades, foi possível extrair uma nota média de satisfação para cada fator da qualidade avaliado. As notas são provenientes das respostas recebidas dos usuários de ônibus transformadas em notas de 0 a 10, através da correspondência apresentada na Figura 2. Para cada cidade são calculadas as notas de satisfação com os 16 fatores da qualidade e a média aritmética das notas desses fatores da qualidade.

\subsubsection{Identificação de potenciais benchmarks}

A literatura (Morpace International e Cambridge Systematics, 1999; Trompet et al., 2013; EQUIP Consortium, 2000) recomenda que a comparação das notas absolutas de pesquisas realizadas em diferentes locais deve levar em conta a chance de fatores externos, como questões culturais e sociais, influenciarem nas avaliações de satisfação, criando vieses. Tendo isso em mente, realizou-se um teste do efeito das variáveis qualitativas "Cidade" (Cidades A, B, C e D) e "Fator da qualidade" (os 16 fatores da qualidade presentes na Pesquisa) através de uma Análise de Variância (ANOVA) de dois fatores sem repetição. A tabela ANOVA completa é apresentada na Tabela 2.

A partir da Tabela 2 pode-se observar que a variável "Cidade" tem efeito significativo nos valores das notas de satisfação dos fatores de qualidade (Valor-P menor do que 5\%), o que comprova que as notas absolutas das percepções dos usuários diferem estatisticamente nas diferentes cidades onde a pesquisa foi aplicada. A comprovação de que a variável "Fator da qualidade" apresenta influência significativa nas notas atribuídas (também teve Valor-P menor do que 5\%) era esperada e não apresenta problemas para as análises, pois cada fator da qualidade será analisado separadamente.

Tabela 2: Tabela ANOVA das notas absolutas de satisfação considerando as variáveis "Cidade" e "Fator da qualidade"

\begin{tabular}{lllllll}
\hline Fonte de Variação & SQ & gl & MQ & F & valor-P & F crítico \\
\hline Fator da qualidade & 49,90 & 15 & 3,13 & 9,54 & $1,725 \mathrm{E}-09$ & 1,89 \\
Cidade & 6,50 & 3 & 2,17 & 6,61 & 0,0008504 & 2,81 \\
Erro & 14,75 & 45 & 0,33 & & & \\
Total & 68,15 & 63 & & & & \\
\hline
\end{tabular}

Com o objetivo de retirar o efeito da variável "Cidade" para reduzir os vieses sociais e culturais, o IBBG propõe uma metodologia de normalização baseada nas notas médias de satisfação de cada cidade (Trompet et al., 2013). A normalização foi realizada descontando-se, da nota de cada fator da qualidade, a média das notas dos fatores da qualidade de cada cidade (conforme Equação 1), obtendo-se as notas normalizadas apresentadas na Tabela 3. 
Tabela 3: Notas de satisfação normalizadas pela média dos fatores da qualidade e por cidade

\begin{tabular}{lllll}
\hline Fatores de qualidade & Cidade A & Cidade B & Cidade C & Cidade D \\
\hline Acesso ao transporte & 1,36 & 0,54 & 1,24 & 1,199 \\
Disponibilidade & 0,79 & $-0,36$ & 0,04 & $-0,49$ \\
Rapidez & 1,43 & 0,76 & 0,19 & 0,26 \\
Confiabilidade & 0,95 & 0,34 & 0,34 & 0,31 \\
Facilidade de fazer transferência & 0,85 & 0,26 & 1,18 & 0,73 \\
Conforto dos pontos de ônibus & $-1,02$ & $-0,99$ & $-1,31$ & $-1,79$ \\
Conforto das estações & $-0,34$ & 0,61 & $-0,54$ & $*$ \\
Conforto dos terminais & $-0,29$ & 0,43 & $-0,27$ & $-0,08$ \\
Conforto dos ônibus & $-0,70$ & 0,85 & $-0,47$ & $-0,72$ \\
Atendimento ao cliente & 0,14 & 0,60 & 1,56 & 1,23 \\
Informação ao cliente & $-0,09$ & 0,37 & 1,16 & 1,36 \\
Segurança pública & $-2,55$ & $-1,23$ & $-2,15$ & $-0,54$ \\
Segurança contra acidentes & $-0,08$ & $-0,09$ & $-0,22$ & 0,25 \\
Exposição a ruído e poluição & $-0,03$ & $-0,36$ & $-0,67$ & $-0,85$ \\
Facilidade em fazer o pagamento & 0,18 & 0,64 & 1,42 & 1,28 \\
Gasto & $-0,60$ & $-2,38$ & $-1,49$ & $-2,13$ \\
Média dos fatores da qualidade & 0,00 & 0,00 & 0,00 & 0,00 \\
\hline
\end{tabular}

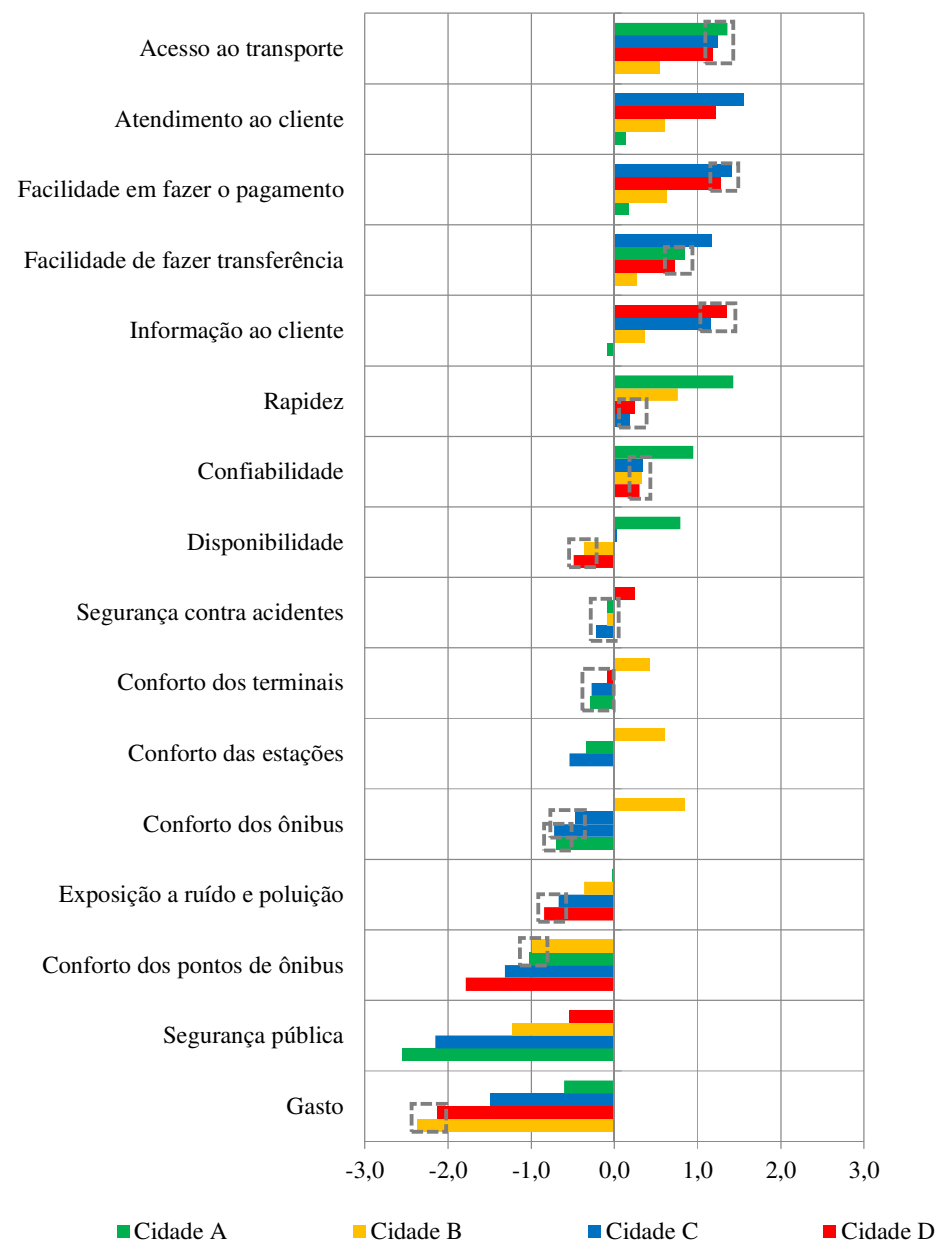

Figura 3. Notas de satisfação normalizadas por fator da qualidade e cidade (retângulos representam notas sem diferença significativa)

Percebe-se que a normalização resulta em notas positivas e negativas, deixando claro quais são os fatores da qualidade avaliados pior, ou melhor, do que a média de cada cidade, ou seja, que contribuem negativa ou positivamente para a boa qualidade do sistema. Além disso, na Tabela 3, observam-se valores 
muito próximos nas avaliações de alguns fatores da qualidade entre as diferentes cidades, o que, dependendo da variabilidade associada a estas notas, não permite concluir com exatidão qual a cidade com as melhores práticas em cada aspecto. Para que se possa definir a cidade que representa o benchmarking em cada fator da qualidade, a partir das notas normalizadas, realizou-se uma Comparação Múltipla de Médias (CMM) para verificar quais notas têm diferença significativa entre si. Os resultados estão plotados no gráfico da Figura 3 que apresenta as notas de satisfação normalizadas para cada fator da qualidade e cidade, conforme legenda de cor. Os retângulos representam as notas sem diferença significativa (Valor-P menor do que 5\%), que caracterizam um empate técnico entre as cidades.

Analisando cada um dos fatores da qualidade em separado e fazendo a comparação entre as cidades, aquelas que possuem o fator de qualidade mais bem avaliados em relação a suas médias provavelmente podem compartilhar uma boa prática com as outras. As cidades que podem ser referência em qualidade para as demais do grupo são chamadas de potenciais benchmarks e são apresentadas na coluna ao lado do gráfico na Figura 3.

Os fatores da qualidade "Exposição a ruído e poluição", “Conforto dos pontos de ônibus", "Segurança pública" e "Gasto" têm todas as notas negativas, ou seja, nenhuma das cidades teve esses aspectos avaliados melhor do que sua própria média. De qualquer modo, as cidades que possuem as maiores notas nesses itens, mesmo que negativas, podem ser investigadas para verificar se têm alguma boa prática que as faz não receberem avaliações tão abaixo da média como as demais, podendo também ser benchmarks.

\subsubsection{Aplicação das análises de benchmarking}

Para ilustrar como as análises de benchmarking podem ser aplicadas ao processo como um todo, a Figura 4 apresenta as notas de satisfação absolutas para cada um dos fatores da qualidade resultantes da aplicação da pesquisa de satisfação na Cidade A. As linhas verticais contínuas correspondem a fatores da qualidade sem diferença significativa entre as notas. Já as estrelas representam as notas de referência para a Cidade A, isto é, as notas que devem ser almejadas por A.

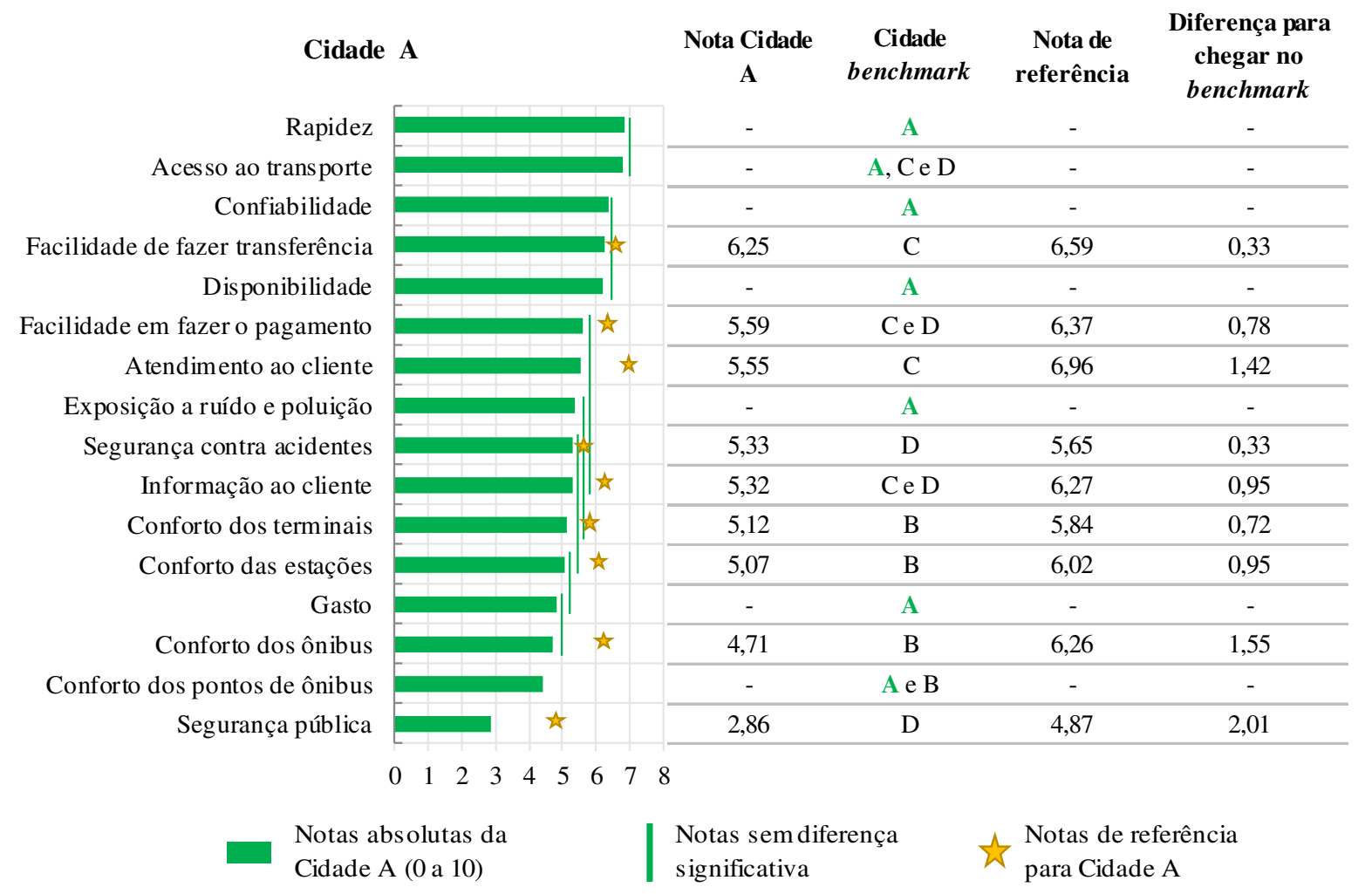

Figura 4. Benchmarking para cidade $\mathrm{A}$ em notas de 0 a 10. (Linhas verticais à direita das barras representam notas sem diferença significativa) 
Inicialmente, buscam-se no gráfico de benchmarking (Figura 3) os potenciais benchmarks para cada um dos fatores da qualidade. As notas de referência para cada fator da qualidade são apresentadas na segunda coluna e representadas pelas estrelas no gráfico da Figura 4. Elas foram calculadas através da soma da nota normalizada da cidade benchmark com a média dos fatores da qualidade da Cidade A. Este método torna a nota absoluta de A, em escala de 0 a 10, comparável com as notas dos potenciais benchmarks. Assim, podem-se identificar, com maior clareza, as metas para a cidade A em cada fator da qualidade. Para os fatores em que o potencial benchmark é a própria cidade A, não se definiu uma meta, pois as análises não mostram outra cidade dentro do grupo que poderia contribuir para qualidade de A.

Com base nessas análises, a priorização de melhorias para a Cidade A deve ser realizada em ordem crescente de notas de satisfação (Figura 4). Neste caso, as análises revelam que o primeiro fator da qualidade candidato a ser priorizado é "Segurança pública", onde a nota da cidade A é 2,86, devendo-se ter como nota de referência 4,87. "Conforto dos pontos de ônibus" seria o segundo fator a ser priorizado, mas a cidade A já é potencial benchmark nesse item. 0 próximo fator da qualidade que pode ser melhorado pelo processo de benchmarking é "Conforto dos ônibus", que possui a cidade B como potencial benchmark, devendo-se atingir a nota de referência de 6,26.

0 processo de priorização dos aspectos que precisam de melhorias pode continuar para que se tenham todos os fatores da qualidade ordenados por priorização. Após essas análises, as próximas etapas do processo de benchmarking são a identificação de boas práticas e troca de experiências entre as cidades, contemplando todas as etapas do processo de benchmarking (American Productivity \& Quality Center, 1996).

É importante perceber que as análises aqui descritas de identificação de potenciais benchmarks e identificação de aspectos prioritários a serem melhorados são ferramentas para nortear o restante do processo de benchmarking. 0 processo deve considerar uma avaliação do contexto de cada cidade e verificar a viabilidade em se aplicar determinadas práticas nos diferentes locais. Assim, devido à complexidade e aos diversos fatores que estão relacionados à melhoria da qualidade de sistemas de transportes, a comunicação e troca de experiências entre as cidades é imprescindível para entender o que pode ser replicado em diferentes locais, o que deve ser adaptado e o que se aplica apenas a um contexto específico.

\section{CONSIDERAÇÕES FINAIS}

Com o objetivo de aplicar análises que permitam a realização de benchmarking com a satisfação dos usuários do transporte coletivo, o presente trabalho verificou que, tanto o instrumento de coleta dos dados, como o método de normalização da satisfação pela média, atenderam às expectativas. A Pesquisa de Satisfação Qualiônibus permite manter um alto nível de padronização da metodologia, o que possibilita a comparação das diferentes aplicações. Já a técnica de normalização da satisfação permite reduzir os vieses culturais e sociais, inerentes a pesquisas de opinião. As análises de benchmarking apresentadas também facilitaram identificar os potencias benchmarks e metas a serem atingidas pelas cidades para os diferentes fatores da qualidade.

Importante ressaltar que, por este trabalho restringir-se ao uso de dados resultantes de aplicações de uma pesquisa específica, o número de sistemas e cidades analisadas foi limitado. Assim, não foi possível agrupar as cidades para que as análises fossem aplicadas apenas a sistemas semelhantes. À medida que se tenha um número maior de cidades, o agrupamento de cidades com perfis similares é indicado, pois fornecerá resultados menos dependentes das análises de contextualização de cada cidade.

Além disso, as análises de variância e de comparação múltipla de médias, realizadas neste trabalho, são fundamentais para validar os resultados obtidos, apesar de muitas vezes serem esquecidas em análises de satisfação. Deve-se sempre ter em mente que, ao trabalhar com percepção dos usuários, são utilizadas pesquisas amostrais e as conclusões devem sempre estar associadas a análises estatísticas.

Importante notar que as análises de benchmarking não identificam de forma absoluta os pontos a serem melhorados e os benchmarks em cada área, mas identificam apenas as áreas e cidades que possuem potencial para tanto. É imprescindível agregar às análises o conhecimento empírico do sistema, 
proveniente da vivência e entendimento das diferentes localidades. Além disso, as análises de benchmarking compreendem apenas uma etapa do processo e não devem estar focadas simplesmente na comparação entre cidades, mas no aprendizado que as cidades podem ter umas com as outras para melhorar a qualidade de seus sistemas de transporte. Para atingir esse objetivo, um grupo de benchmarking deve ter seus membros ativos, promover encontros, facilitar o contato e a troca de experiência, além de ser um processo contínuo de melhoria. É o processo como um todo que faz do benchmarking uma boa ferramenta de melhoria da qualidade e o motivo pelo qual se disseminou globalmente.

\section{REFERÊNCIAS}

Alouche, P. L. e Barbosa, M. C. (1997) Benchmarking: um importante projeto do metrô de São Paulo. Revista dos transportes públicos, ANTP, n. 77, p. 63-72.

American Productivity \& Quality Center - APQC (1996) Emerging Best Practices in Knowledge Management, American Productivity \& Quality Center, Houston, TX.

Bertozzi, P. P. e O. F. Lima Jr. (1998) A qualidade no serviço de transporte público sob as óticas do usuário, do operador e do órgão gestor. Revista dos Transporte Públicos, n. 081, p. 53-66.

Bhutta K. S. e Huq F. (1999) Benchmarking - Best practices: as integrated approach. Benchmarking: An International Journal, v. 6, iss 3, p. 254-268. DOI: 10.1108/14635779910289261

EMBARQ Brasil (2014) Qualiônibus pesquisa de Satisfação. EMBARQ Brasil, Porto Alegre. Disponível em: <http://embarqbrasil.org/node/47381> Acesso em: 04 de jul 2016.

EMTA (2016) BEST: Benchmarking of customer satisfaction with public transport in Europe. Disponível em: http://www.emta.com/spip.php?article668\&lang=en Acesso em: 04 jul 2016.

EQUIP Consortium (2000). Deliverable 5 - Final Report - Project EQUIP Contract No. UR-98-RS.3076, Disponível em: <http://www.transport-research.info/sites/default/files/project/documents/equip.pdf> Acesso em: 25 de out. 2017.

European Commission. Quality approach in tendering urban public transport operations. Transport Research Fourth Framework Programme Urban Transport - Report 76, 229 p., European Communities, Luxembourg.

European Standard (2002) EN 13816: transportation - logistics and services - public passenger transport - service quality definition, targeting and measurement. Bruxelas.

Ferraz, A. C. P. e Torres, I. G. E. (2004) Transporte Público Urbano. 2. ed. ampl. e atual. São Carlos.

Kittelson \& Associates, Inc; Urbitan, Inc; C. S. LKC, Inc; I. Morpace e U. of T. Queensland (2003) A Guidebook for Developing a Transit Performance-Measurement System - Report 88. Washington, DC.

Kittelson \& Associates, Inc. (2003) Transit Capacity and Quality of Service Manual. Transit Cooperative Research Program: Report 100 (2nd ed.). Washington, DC, USA.

Landry, P. (1993) Benchmarking strategy. Executive Excellence, v. 10, iss. 6, p. 8-9.

Likert, R. (1932) A technique for the measurement of attitudes. The Science Press: New York.

Morpace International, Inc. e Cambridge Systematics, Inc. (1999) A Handbook for Measuring Customer Satisfaction and Service Quality - Report 47. Washington, DC.

Oliveira E. F. T. da e M. C. C. Grácio (2005) Análise a respeito do tamanho de amostras aleatórias simples: uma aplicacão na área de Ciência da Informacão. Revista de Ciência da Informação, v. 6, n. 3.

Randall, E. R., B. J. Condry, e M. Trompet (2007) International Bus System Benchmarking: Performance Measurement Development, Challenges, and Lessons Learned. In Transportation Research Board 86th Annual Meeting, TRB, 2007.

Ribeiro, G. R. S. B. e R. C. Gonçalves (2015) Estruturação de metodologia de benchmarking para o setor de transporte rodoviário de passageiros. In XXIX Congresso Nacional de Pesquisa em Transporte da ANPET. Ouro Preto, nov 2015.

Ribeiro, J. L. D.; Echeveste, M. E.e A. M. F Danilevicz. (2001) A utilização do QFD na otimização de produtos, processos e serviços. Porto Alegre: FEENG/UFRGS. Série Monográfica Qualidade.

Souza, F. B. B. e F. D. Michel (2007) Avaliação segmentada do sistema de transporte público: uma análise qualitativa para tomada de decisão. Anais do XXI Congresso de Pesquisa e Ensino em Transportes, ANPET, Rio de Janeiro.

Trompet, M.; R. Parasram e R.J. Anderson (2013) Benchmarking disaggregate customer satisfaction scores between bus operators in different cities and countries. In Transportation Research Record: Journal of the Transportation Research Board, v. 2351, p. 14-22, DOI:10.3141/2351-02

Zairi, M. (1996) Effective Benchmarking: learning from the best. Chapman \& Hall, London. 